ESAIM: PROCEEDINGS AND SURVEYS, September 2014, Vol. 45, p. 158-167

J.-S. Dhersin, Editor

\title{
CONTINUOUS-IN-TIME FINANCIAL MODEL FOR PUBLIC COMMUNITIES *
}

\author{
Emmanuel FrÉnod ${ }^{1}$ And Mohamad SAFA ${ }^{2}$
}

\begin{abstract}
The achievement of a project requires tools to monitor and adjust its evolution over time. Rather than to check at mid-term whether the objectives will be achieved or not, and adjust them, it is interesting to develop a control tool in order to effectively conduct the project's objectives. In this paper, we improve the continuous-in-time financial model developed in Frénod \& Chakkour [2], that describes working of loan and repayment, in order to prepare its capability to be used in control theory approach. The aim of this study is to determine the optimal loan schedule taking into account the objective of the project, the income and the spending. For that, we set out an optimal control method for the strategy elaboration phase to better adjust the project implementation.
\end{abstract}

Résumé. La réalisation d'un projet nécessite des outils pour surveiller et ajuster son évolution au fil du temps. Plutôt que de vérifier à mi-parcours si les objectifs seront atteints ou non, et les adapter, il est intéressant de développer un outil de contrôle afin de mener efficacement les objectifs du projet. Dans cet article nous adaptons le modèle financier continu en temps, développé par Frénod \& Chakkour [2] et qui décrit la façon d'emprunter et de rembourser, afin de l'utiliser dans le cadre de la théorie de contrôle. Le but c'est de déterminer la stratégie d'emprunt optimal pour atteindre les objectifs d'un projet. Cette stratégie doit tenir compte du délai du projet, du taux d'intérêt de l'emprunt, des recettes et des dépenses courantes. Une fois cette stratégie est établie, et tout au long du déroulement du projet, il est indispensable de contrôler la façon dont le plan de l'emprunt est implémenté. Pour cela, nous avons mis en place une méthode de contrôle optimal de la phase d'élaboration de la stratégie, afin de mieux ajuster la mise en œuvre de l'implémentation du projet.

\section{INTRODUCTION}

A project is a temporary activity group designed to produce a product, service or result. A project is temporary in that sense it has a defined beginning and end in time and so a lifetime. When the project involves a loan, it is necessary to find the best strategy in order to achieve the goals of the project, minimizing the cost of the loan. This strategy should be able to achieve the project goals on time under constraints that may be imposed by law, taxes, banking institutions and more generally by the project environment. It gives rise to the loan scheme which is the way that the amounts concerned by the loan will be borrowed.

Once this strategy set out, and all along the project implementation, it is needed to control the way the loan scheme is implemented. The objective is to counterbalance the gap between the reality of the environment in which the project takes place and the forecast made during the strategy elaboration.

\footnotetext{
* This work is jointly funded by MGDIS company (http://www.mgdis.fr/) and the PEPS program Labex AMIES (http://www.agence-maths-entreprises.fr/)

1 Univ. Bretagne-Sud, UMR 6205, LMBA, F-56000 Vannes, France

2 Univ. Bretagne-Sud, UMR 6205, LMBA, F-56000 Vannes, France
}

(C) EDP Sciences, SMAI 2014 
There are several references in the literature dealing with continuous-in-time financial model. Among them we find: R. Merton [5] which provides an overview and synthesis of finance theory from the perspective of continuous-in-time analysis. In [7], S. Sundaresan surveys and assesses the development of continuous-in-time methods in finance during the period between 1970 and 2000. In addition, many studies have used control engineering methods and techniques in finance. For example Keel [4] explored and extended optimal portfolio construction techniques currently found in the literature. Grigorieva \& Khailov [3] built a controlled system of differential equations modeling a firm that takes a loan in order to expand its production activities.

In this paper we base on a continuous-in-time financial model developed in Frénod \& Chakkour [2] that describes working of loan and repayment. This model is not designed for the financial market but for the public institutions. The main objective of this paper is to use this model in the framework of the control theory. For that, we set out an optimal control method for the strategy elaboration phase and an adaptive control method to better adjust the project implementation.

\section{Continuous-in-time financial model}

In this section we improve the continuous-in-time financial model of Frénod \& Chakkour [2] in order to account for the project notion and to prepare its capability to be used in control theory approach. The time domain is the interval $[0, \Theta]$, where $\Theta>0$ is the lifetime of the project. We consider that beyond $\Theta$ the spending associated with the project are done, the loan associated with the project is completely paid off and the project is finished.

\subsection{Variables of the model}

To characterize the budget of a project, we introduce the loan density $\kappa_{E}$ and the density of repayment $\rho_{K}$ which is connected, as explained in Frénod \& Chakkour [2], to the loan density by a convolution operator:

$$
\rho_{K}(t)=\left(\kappa_{E} * \gamma\right)(t)
$$

where $\gamma$ is the repayment pattern. Since the whole amount associated with the loan has to be repaid, $\gamma$ has to satisfies:

$$
\int_{-\infty}^{+\infty} \gamma(t) d t=1
$$

We denote by $\mathcal{K}_{R D}$ the current debt, given as the solution to the following differential equation:

$$
\frac{d \mathcal{K}_{R D}(t)}{d t}=\kappa_{E}(t)-\rho_{K}(t)-\rho_{K}^{\mathcal{I}}(t)
$$

where $\rho_{K}^{\mathcal{I}}(t)$ is the density of repayment of the current debt $\mathcal{K}_{R D}(0)$ at the beginning of the period. It is called initial debt repayment scheme. Initial condition for equation (3) is given by:

$$
\mathcal{K}_{R D}(0)=\int_{0}^{+\infty} \rho_{K}^{\mathcal{I}}(t) d t
$$

We denote by $\rho_{I}(t)$ the density of interest defined by:

$$
\rho_{I}(t)=\alpha(t) \mathcal{K}_{R D}(t)
$$

where $\alpha(t)$ is the floating rate interest. The algebraic spending density is denoted $\sigma(t)$, it takes into account the spending and the income and it is given by:

$$
\sigma(t)=\beta(t)+\sigma_{g}(t)
$$


where $\beta(t) \geq 0$ is the "financing needs density", it is the density of spending that are intended for the project only. $\sigma_{g}(t)$ is the current spending density. We assume that $\beta(t) \geq 0$ because only spending are concerned.

The fact that the initial time of the project is 0 and the lifetime is $\Theta$ translates as:

$$
\operatorname{supp}\left(\kappa_{E}\right) \subset[0, \Theta], \quad \operatorname{supp}\left(\kappa_{E}\right)+\operatorname{supp}(\gamma) \subset[0, \Theta], \quad \operatorname{supp}\left(\rho_{K}^{\mathcal{I}}\right) \subset[0, \Theta],
$$

where supp $(f)$ is the support of $f$.

\subsection{Objectives of the project}

Integrating (3) over $[0, t]$, we obtain using (1) the following relation:

$$
\mathcal{K}_{R D}(t)=\mathcal{K}_{R D}(0)+\int_{0}^{t}\left(\kappa_{E}-\kappa_{E} * \gamma\right)(s) d s-\int_{0}^{t} \rho_{K}^{\mathcal{I}}(s) d s,
$$

and using (4), we obtain:

$$
\mathcal{K}_{R D}(t)=\int_{0}^{t}\left(\kappa_{E}-\kappa_{E} * \gamma\right)(s) d s+\int_{t}^{\infty} \rho_{K}^{\mathcal{I}}(s) d s .
$$

We want that the spending density balances the income density. In our model we have the following densities: $\sigma$ which, depending on its sign, stands alternately for income or spending, $\kappa_{E}$ which is an income density and $\rho_{K}, \rho_{I}, \tilde{\rho}_{K}^{\mathcal{I}}$ which are spending densities. Hence the balance relation reads:

$$
\sigma(t)=\kappa_{E}(t)-\rho_{K}(t)-\rho_{I}(t)-\rho_{K}^{\mathcal{I}}(t)
$$

Using (9) and (5), we deduce the following relation:

$$
\begin{aligned}
\sigma(t) & =\kappa_{E}(t)-\left(\kappa_{E} * \gamma\right)(t)-\alpha(t) \mathcal{K}_{R D}(t)-\rho_{K}^{\mathcal{I}}(t) \\
& =\left(\mathcal{L}\left[\kappa_{E}\right]\right)(t)-\alpha(t) \int_{t}^{\infty} \rho_{K}^{\mathcal{I}}(s) d s-\rho_{K}^{\mathcal{I}}(t),
\end{aligned}
$$

where the operator $\mathcal{L}$ is defined by:

$$
\left(\mathcal{L}\left[\kappa_{E}\right]\right)(t) \doteq \kappa_{E}(t)-\left(\kappa_{E} * \gamma\right)(t)-\alpha(t) \int_{0}^{t}\left(\kappa_{E}-\kappa_{E} * \gamma\right)(s) d s
$$

is the algebraic income density associated to the loan. In other words, it is the difference between the income density induced by the loan density on the one hand and the spending density associated with the repayment density and the interest payment density on the other hand. Using (6) we have then:

$$
\beta(t)=\left(\mathcal{L}\left[\kappa_{E}\right]\right)(t)-\alpha(t) \int_{t}^{\infty} \rho_{K}^{\mathcal{I}}(s) d s-\rho_{K}^{\mathcal{I}}(t)-\sigma_{g}(t) .
$$

The isolated spending density $\beta(t)$ is the difference between the algebraic income density associated with the loan, and the spending densities related to the following: current spending, initial debt repayment and payment of the interests of this latter.

We define an objective as a couple collection $\left(c_{i}, \Theta_{i}\right), i \in\{1, \ldots, N\}$, where $c_{i}$ is the amount which has to be spent for the project at the moment $\left.\Theta_{i} \in\right] 0, \Theta\left[\right.$. We suppose that $0<\Theta_{1}<\Theta_{2}<\ldots<\Theta_{N}<\Theta$, and to be consistant we need that $0 \leq c_{1} \leq c_{2} \leq \ldots \leq c_{N}$. We say that the objective is reached if:

$$
\int_{0}^{\Theta_{i}} \beta(t) d t \geq c_{i}, \forall i=1, \ldots, N
$$


The above equation indicating that at any $\Theta_{i}$ the amount allocated to the project is at least the amount needed for the project.

Using this model we will establish the strategy, i.e. find the loan which allows the objectives $\left(c_{i}, \Theta_{i}\right)$ to be reached. Furthermore, this loan is not chosen at random but have to satisfy some conditions. Typically, it must minimize the cost of the loan. This strategy can be written as an optimal control problem which is developed in the next section.

\section{Strategy Elaboration: optimize LOAN COST}

Among all the variables introduced in the previous section, the only one that can be modified is the loan density $\kappa_{E}$. We consider that the repayment pattern $\gamma$ and the interest rate $\alpha$ are essentially imposed by banking institutions. The density of algebraic spending $\sigma$ is imposed by the project environment. Hence $\gamma, \alpha$ and $\sigma$ are, at the level of the strategy elaboration, foresights of what they will be when the project will be implemented.

We introduce the loan cost which is induced by interest payments $C_{I}\left[\kappa_{E}\right]$ and which is defined as:

$$
C_{I}\left[\kappa_{E}\right]=\int_{0}^{\infty}\left(\rho_{I}\left[\kappa_{E}\right]\right)(t) d t
$$

where $\boldsymbol{\rho}_{\boldsymbol{I}}$ is the functional that links loan density $\kappa_{E}$ to density of interest $\rho_{I}$ :

$$
\left(\boldsymbol{\rho}_{\boldsymbol{I}}\left[\kappa_{E}\right]\right)(t)=\alpha(t) \mathcal{K}_{R D}(t)=\alpha(t)\left(\int_{0}^{t}\left(\kappa_{E}-\kappa_{E} * \gamma\right)(s) d s+\int_{t}^{\infty} \rho_{K}^{\mathcal{I}}(s) d s\right)
$$

and the total amount of the loan $C_{E}\left[\kappa_{E}\right]$ is defined by:

$$
C_{E}\left[\kappa_{E}\right]=\int_{0}^{\infty} \kappa_{E}(t) d t
$$

Minimizing loan cast consists in finding loan density $\kappa_{E}$ that minimizes the loan cost $C_{I}\left[\kappa_{E}\right]$.

We introduce now the following two optimization problems in a intuitive and not mathematically completely rigorous way. Those problems called (O1) and (O2) will then be inserted within rigorous framework in the next section.

\subsection{O1. Optimization of the spending.}

Given $\left(c_{i}, \Theta_{i}\right), i \in\{1, \ldots, N\}$, find the loan density $\breve{\kappa}_{E}$ solution to:

$$
C_{I}\left[\breve{\kappa}_{E}\right]=\min _{\kappa_{E}} C_{I}\left[\kappa_{E}\right]
$$

subject to:

$$
\int_{0}^{\Theta_{i}} \beta(t) d t \geq c_{i}, \forall i=1, \ldots, N .
$$

In this problem we seek the optimal loan density $\breve{\kappa}_{E}(t)$ that minimizes the cost of the loan, considering that the amount allocated to the project until $\left.\Theta_{i} \in\right] 0, \Theta\left[\right.$ is at least the amount $c_{i}$ needed for the project at $\Theta_{i}$. There is no uniqueness of the solution to problem (O1). Among its solution of problem (O1) some are subjected to perturbations which are numerical artifices that are irrelevant from financial slant. We will therefore build a method based on filtering theory to eliminate those perturbations. Several filters will be investigated in next section to choose a suitable one to be incorporated in our method. We introduce here the general framework of the filtering based problem which is set in terms of an optimization problem. 


\subsection{O2. Filtering perturbed solutions to (O1) by an optimization problem.}

From all solutions $\breve{\kappa}_{E}(t)$ resulting from (O1), we denote by $\hat{\kappa}_{E}(t)$ the desired unperturbed solution obtained by eliminating the perturbations in $\breve{\kappa}_{E}(t)$, which is defined by:

$$
\hat{\kappa}_{E}(t)=\breve{\kappa}_{E}(t)-\hat{G}(t)
$$

where $\hat{G}(t)$ represents the perturbations density. $\hat{G}(t)$ is obtained as the result of the following optimization problem (called (O2)) which can be written generically as: given $\breve{\kappa}_{E}(t)$, find $\hat{G}(t)$ solution of:

$$
\min _{G} F\left(\breve{\kappa}_{E}, G\right)
$$

where the definition of the functional $F$ is given in Section (4.1) and depends on the filter which is used. The problem (19a) is subject to the following constraints:

$$
\int_{0}^{\Theta_{i}} \beta(t) d t \geq c_{i}, \forall i=1, \ldots, N
$$

Moreover, since $G$ is the perturbation to be removed, it satisfies:

$$
\int_{0}^{\Theta} G(t) d t=0
$$

This is then a supplementary constraint for optimization problem (O2).

\section{Optimization problem (O1)}

\subsection{Mathematical framework of optimization problem (O1)}

The objective of this section is to build the mathematical framework of the optimal control problem. We suppose that $\kappa_{E}(t), \gamma(t)$ and $\rho_{K}^{\mathcal{I}}(t)$ introduced in section 1.1 are in $L^{2}([0, \Theta])$ and $\alpha(t), \mathcal{K}_{R D}(t)$ are in $L^{\infty}([0, \Theta])$. Let $U$ be the set of admissible loan densities $\kappa_{E}(t) \in L^{2}[0, \Theta]$, such that $\kappa_{E}^{\max }(t) \geq \kappa_{E}(t) \geq 0$, where $\kappa_{E}^{\max }(t) \in$ $L^{\infty}([0, \Theta])$ is the maximal loan densities that can be borrowed. The admissible loan density should allow to have a positive financing needs:

$$
\beta(t) \geq 0 .
$$

By using (13), equation (20) is equivalent to write:

$$
\left(\mathcal{L}\left[\kappa_{E}\right]\right)(t) \geq \sigma_{g}(t)+\alpha(t) \int_{t}^{\infty} \rho_{K}^{\mathcal{I}}(s) d s+\rho_{K}^{\mathcal{I}}(t),
$$

and equation (17b) can be written in the next form:

$$
\int_{0}^{\Theta_{i}}\left(\mathcal{L}\left[\kappa_{E}\right]\right)(t) d t \geq c_{i}+\int_{0}^{\Theta_{i}}\left[\alpha(t) \int_{t}^{\infty} \rho_{K}^{\mathcal{I}}(s) d s+\rho_{K}^{\mathcal{I}}(t)+\sigma_{g}(t)\right] d t, \forall i=1, \ldots, N .
$$

We can rewrite the optimization problem in a rigorous form. Denoting $U$ the set of function $\kappa_{E}(t) \in L^{2}[0, \Theta]$ which satisfy the conditions: 


$$
\begin{aligned}
\kappa_{E}^{\max } & \geq \kappa_{E}(t) \geq 0 \\
\left(\mathcal{L}\left[\kappa_{E}\right]\right)(t) & \geq \mu(t) \\
\int_{0}^{\Theta_{i}}\left(\mathcal{L}\left[\kappa_{E}\right]\right)(t) d t & \geq c_{i}+\int_{0}^{\Theta_{i}} \mu(t) d t, \forall i=1, \ldots, N,
\end{aligned}
$$

where

$$
\mu(t)=\sigma_{g}(t)+\alpha(t) \int_{t}^{\infty} \rho_{K}^{\mathcal{I}}(s) d s+\rho_{K}^{\mathcal{I}}(t),
$$

we search a solution $\kappa_{E}(t)$ of the following optimization problem :

$$
C_{I}\left[\breve{\kappa}_{E}\right]=\min _{\kappa_{E} \in U} C_{I}\left[\kappa_{E}\right] .
$$

It is recalled that functions $\sigma_{g}(t), \rho_{K}^{\mathcal{I}}(t), \alpha(t)$ and $\gamma(t)$ are considered as known and are model inputs.

From now on, we work with a fixed interest rate $\alpha$, in this case we can show that solving the problem (25) is equivalent to solve the following problem:

$$
C_{E}\left[\breve{\kappa}_{E}\right]=\min _{\kappa_{E} \in U} C_{E}\left[\kappa_{E}\right]
$$

which means: minimize the cost of the loan is equivalent to minimize the total amount of the loan. The latter is the problem that we will be interested in. We have the following lemma:

Lemma 1. For a fixed $\alpha \geq 0$, each function $\kappa_{E} \in L^{2}([0, \Theta])$ solution of (25) is also solution of (26) and vice versa.

\subsection{Existence of solutions for optimization problem (O1)}

In this section we show the existence of solutions for optimization problem (26). By introducing the following notation:

$$
\mathcal{F}_{g} \doteq\left\{f \in L^{2}[0, \Theta] ; f \geq g\right\}, \text { for } g \in L^{2}[0, \Theta],
$$

and the continuous functionals $\Psi_{i}, i=1, \ldots, N$, defined by:

$$
\begin{aligned}
\Psi_{i}: L^{2}[0, \Theta] & \rightarrow \mathbb{R} \\
f & \longmapsto \Psi_{i}(f)=\int_{0}^{\Theta_{i}} \mathcal{L}(f(t)) d t,
\end{aligned}
$$

we can then rewrite $U$ in the next form:

$$
U=\mathcal{F}_{0} \cap-\mathcal{F}_{-\kappa_{E}^{\max }} \cap \mathcal{L}^{-1}\left(\mathcal{F}_{\mu(t)}\right) \cap\left[\bigcap _ { i = 1 } ^ { N } \Psi _ { i } ^ { - 1 } \left(\left[c_{i}+\int_{0}^{\Theta_{i}} \mu(s) d s,+\infty[)\right] .\right.\right.
$$

We will invoke the following theorem which proof can be found in Cohen [1].

Theorem 2. Let $V$ be a subspace of a Hilbert space. If $J: V \mapsto \overline{\mathbb{R}}$ is a lower semi-continuous function, convexe and coercive on $V$, if $V$ is convexe and closed, if

$$
\operatorname{Dom}(J) \cap V \neq \emptyset \text {, where } \operatorname{Dom}(J)=\{x \in V ; J(x)<+\infty\},
$$

then there exists at least a solution of the optimization problem: $\min _{x \in V} J(x)$. 
Using Theorem 2 we obtain the next theorem:

Theorem 3. If set $U$ given by (29) is such that $U \neq \emptyset$, then optimization problem (26) has at least one solution.

Proof. The set $U$ given by (29) is convex and closed because it is the intersection of closed sets. In fact $\mathcal{F}_{g}$ is a closed set, $\mathcal{L}$ is a continuous linear operator and $\Psi$ is a continuous operator. The functional:

$$
\begin{aligned}
C_{E}: L^{2}[0, \Theta] & \rightarrow \mathbb{R} \\
f & \longmapsto C_{E}[f]=\int_{0}^{\Theta} f(t) d t,
\end{aligned}
$$

is a continuous function. In addition, $C_{E}$ is a convex function because it is linear on $U$ and coercive because $U$ is a bounded set (constraint (23a)). Since $\operatorname{Dom}\left(C_{E}\right)=L^{2}([0, \Theta])$ and according to Theorem 2, if $U \neq \emptyset$ then optimization problem (26) has at least one solution.

\section{Optimization problem (O2)}

\subsection{Mathematical framework of optimization problem (O2)}

In this section we build a mathematical framework for the optimization problem (O2) introduced in Section 2. We suppose the optimal loan density $\breve{\kappa}_{E}(t)$ and the perturbation density $\hat{G}(t)$ are in $L^{2}([0, \Theta])$. The functional $F$ introduced in Section 2 is a map from $L^{2}[0, \Theta] \times L^{2}[0, \Theta]$ to $\mathbb{R}$. Having $\breve{\kappa}_{E}(t)$ resulting from (O1), we search $\hat{G}(t)$ solution of the next optimization problem. Given $\breve{\kappa}_{E}(t)$, find $\hat{G}(t)$ solution of:

$$
\min _{G} F\left(\breve{\kappa}_{E}, G\right)
$$

subjected to the same constraints of (O1), where $\kappa_{E}(t)$ is replaced by $\breve{\kappa}_{E}(t)-G(t)$ in formulas (23), and to constraint (19c). We can rewrite this optimization problem called (O2) in a rigorous form. Denoting $U_{2}$ the set of function $G \in L^{2}([0, \Theta])$ which satisfy the conditions:

$$
\begin{aligned}
\kappa_{E}^{\max } & \geq \breve{\kappa}_{E}(t)-G(t) \geq 0, \\
\left(\mathcal{L}\left[\breve{\kappa}_{E}-G\right]\right)(t) & \geq \mu(t), \\
\int_{0}^{\Theta_{i}}\left(\mathcal{L}\left[\breve{\kappa}_{E}-G\right]\right)(t) d t & \geq c_{i}+\int_{0}^{\Theta_{i}} \mu(t) d t, \forall i=1, \ldots, N, \\
\int_{0}^{\Theta} G(t) d t & =0 .
\end{aligned}
$$

where $\mu(t)$ is defined by (24). For given $\breve{\kappa}_{E}(t)$ obtained from the optimization problem (O1), we search $\hat{G}(t)$ solution of the next optimization problem:

$$
\min _{G \in U_{2}} F\left(\breve{\kappa}_{E}, G\right)
$$

and we thus obtain the unperturbed solution $\hat{\kappa}_{E}(t)=\breve{\kappa}_{E}(t)-\hat{G}(t)$. The type of filter that we will use depends on the choice of $F$. We will investigated two types of filters: the first one is based on the thresholding method and the second on the total variation denoising (TVD).

\subsubsection{Thresholding methode.}

It consists in eliminating the low and very high frequency of a signal, by writing the functional $F$, for all $Y$ and $G$ in $L^{2}[0, \Theta]$, as the following form:

$$
F(Y, G)=\lambda_{1} \int_{-\infty}^{+\infty}|G(t)|^{2} d t+\lambda_{2} \int_{-\infty}^{+\infty}\left|\mathcal{F}^{-1}\left(\mathcal{F}\left(\frac{\partial(Y-G)}{\partial t}\right) \rho_{\eta}\right)\right| d t
$$


where $\mathcal{F}$ is the Fourier transform operator and for fixed $\eta>0, \rho_{\eta}(t)=0$ if $|t|>\eta$. The second member of (34) collects the mid-frequency of the derivative of $(Y-G)(t)$.

\subsubsection{Total Variation Denoising (TVD).}

It is a tool for noise reduction developed so as to preserve sharp edges in the underlying signal [6]. In such cases we can define the functional $F$ as:

$$
F(Y, G)=\lambda_{1} \int_{-\infty}^{+\infty}|G(t)|^{2} d t+\lambda_{2} \int_{-\infty}^{+\infty}\left|\frac{\partial(Y-G)}{\partial t}\right| d t
$$

In (34) and (35), $\lambda_{1}>0$ and $\lambda_{2}>0$ are two regularization parameters to control the degree of smoothing. Increasing $\lambda_{2}$ gives more weight to the second term which measures the total variation of $(Y-G)(t)$.

Since the function $F(Y, G)$ is strictly convex for a fixed $Y$ and the set $U_{2}$ defined by (32) is convex, then we have the uniqueness of the solution of the optimization problem $(\mathrm{O} 2)$.

\section{Numerical Simulations}

We solve the optimization problem (26) by using the simplex method. The numerical simulations were performed by using Matlab. We impose the repayment pattern $\gamma$, the current spending $\sigma_{g}$ and we seek the optimal loan $\breve{\kappa}_{E}$ which allows the objectives of the project to be realized.

\subsection{Example 1}

In Example 1, the repayment is done in a constant way between the first and 6th year after borrowing. We pay off nothing outside this period. The current spending density $\sigma_{g}$ alternates between positive values, corresponding to periods where income is less than spending and negative values, corresponding to periods where income is larger than spending. The objective to be achieved are fixed at the end of the 4nd and 14th year. The optimal loan density is given in Figure 1. As we can see on the right side of Figure 1 the constraints are satisfied. For this example we have taken:

$$
\Theta=20, \quad n=1000, \quad \alpha=0.01, \quad \rho_{K}^{\mathcal{I}}=0, \quad \Theta_{1}=4, \quad \Theta_{2}=14, \quad c_{1}=6, \quad c_{2}=17 .
$$

Figure 1 on the right shows the isolated spending density $\beta(t)$ and the isolated spending $\int_{0}^{\Theta} \beta(t) d t$ obtained from Example 1. As we can see, we get $\beta(t) \geq 0$ and $\int_{0}^{\Theta_{i}} \beta(t) d t \geq c_{i}, i=1,2$. This means that the constraints of the optimization problem (O1) are satisfied and the project objectives are reached.

\subsection{Example 2}

In Example 2, the repayment is only made in an increasing way between the 7th and 11th year after borrowing. We pay off nothing outside this period. The optimal loan density is given in Figure 2. For this example we have taken:

$$
\Theta=20, \quad n=1000, \quad \alpha=0.01, \quad \rho_{K}^{\mathcal{I}}=0, \quad \Theta_{1}=10, \quad \Theta_{2}=12, \quad c_{1}=15, \quad c_{2}=20 .
$$

Figure 2 on the right shows the isolated spending density $\beta(t)$ and the isolated spending $\int_{0}^{\Theta} \beta(t) d t$ obtained from Example 1. As we can see, we get $\beta(t) \geq 0$ and $\int_{0}^{\Theta_{i}} \beta(t) d t \geq c_{i}, i=1,2$. This means that the constraints of the optimization problem (O1) are satisfied and the project objectives are reached. 

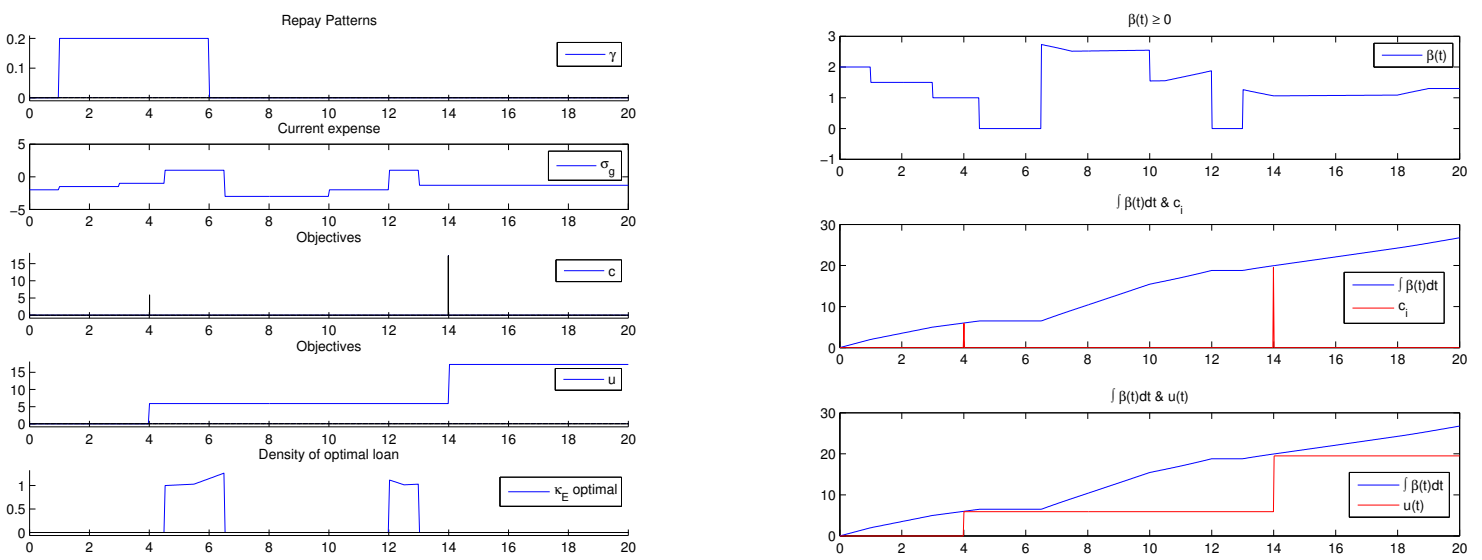

FiguRE 1. On the left we have the optimal loan $\breve{\kappa}_{E}$ obtained for given: repayment pattern $\gamma$, objectives $c$ and current spending $\sigma_{g}$ (for Example 1). On the right we check if the constraints are verified (for Example 1)
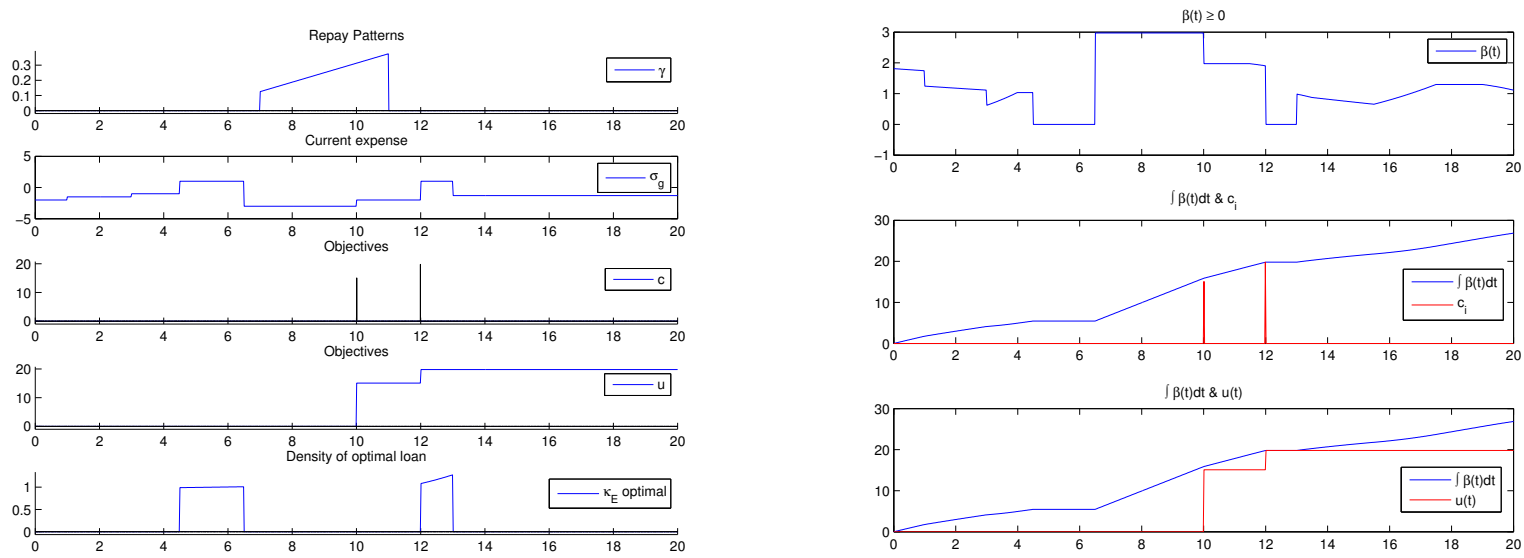

FiguRE 2. Optimal loan $\kappa_{E}$ obtained for given: repayment pattern $\gamma$, objectives $c$ and current spending $\sigma_{g}$.

\section{REFERENCES}

[1] G. Cohen. Convexité et optimisation. Cours, 2000.

[2] E. Frénod and T. Chakkour. A continuous-in-time financial model. Work in progress, 2013.

[3] E.V. Grigorieva and E.N. Khailov. Optimal control of a commercial loan repayment plan. Discret and continuous dynamical systems, pages 345-354, 2005.

[4] S.T. Keel. Optimal Portfolio Construction and Active Portfolio Management Including Alternative Investments. Phd thesis, ETH Zurich, 2006.

[5] R.C. Merton. Continuous-Time Finance. Blackwell, 1st edition, 1992.

[6] L. Rudin, S. Osher, and E. Fatemi. Nonlinear total variation based noise removal algorithms. Physica D: Nonlinear Phenomena, 60(1-4):259-268, 1992. 
[7] S.M. Sundaresan. Continuous-time methods in finance: A review and an assessment. Journal of Finance, pages 1569-1622, 2000. 